\title{
Dependence of relational colour constancy on the extraction of a transient signal
}

\author{
Karina J Linnell $\mid$, David H Foster\# \\ Department of Communication and Neuroscience, Keele University, Staffordshire ST5 5BG, UK \\ Received 18 July 1995, in revised form 22 December 1995
}

\begin{abstract}
Human observers can correctly attribute changes in the appearance of a scene either to changes in the incident light or to changes in the spectral-reflectance properties of the scene. This ability was assessed as a function of the time course of illuminant and spectral-reflectance changes. Observers were presented with computer simulations of Mondrian patterns of 49 randomly selected Munsell papers. On each trial a Mondrian pattern was presented for $1 \mathrm{~s}$; the pattern then changed either instantaneously or gradually into another Mondrian pattern, also presented for $1 \mathrm{~s}$, which was related to the first either by an illuminant change or by an illuminant change accompanied by additional changes in the spectral-reflectance functions of the individual papers. Illuminant and spectral-reflectance changes were applied linearly in time (with respect to CIE coordinates) over intervals ranging from 0 to $7 \mathrm{~s}$. Observers indicated whether there was a spectral-reflectance change. They were able to make reliable discriminations between illuminant and spectral-reflectance changes both when the changes were applied instantaneously and when they were applied gradually over time, but performance worsened progressively as the duration of the changes increased, that is, as their rate decreased. It is suggested that discrimination in this task depends on the extraction of a low-level transient signal which is generated in response to rapid changes in scene appearance and which is progressively attenuated as changes occur more and more gradually.
\end{abstract}

\section{Introduction}

'Colour constancy' refers to the constancy of the perceived colours of surfaces in a scene despite changes in the illuminant upon it. When measured by colour-matching methods the extent of this constancy in human observers seems limited (see eg Arend and Reeves 1986; Tiplitz Blackwell and Buchsbaum 1988; Valberg and Lange-Malecki 1990; Troost and de Weert 1991; Reeves 1992; Bramwell and Hurlbert 1993; Lucassen and Walraven 1993; Nascimento 1995; Cornelissen and Brenner 1995; Nascimento and Foster 1996a). In a formal sense, the traditional approach to colour constancy can be interpreted in terms of the ability to discriminate illuminant changes on a scene from changes in the spectral reflectances of the surfaces making up the scene (Foster and Nascimento 1994, appendix 1). The latter ability has been successfully demonstrated in human observers (Craven and Foster 1992).

In general, the change in appearance which ensues when an illuminant change occurs is spatially uniform, whereas that which ensues when a spectral-reflectance change occurs is spatially nonuniform. It has therefore been assumed that the ability to make discriminations between illuminant and spectral-reflectance changes is based on a visual coding of spatial colour relations within a scene, such that only when these relations are preserved ('relational colour constancy') to within certain limits can a change in appearance be attributed to an illuminant change rather than to a spectralreflectance change (Foster and Nascimento 1994).

Craven and Foster (1992) have shown that when illuminant and spectral-reflectance changes occur effectively instantaneously they can be discriminated from one another (compare Foster et al 1992). In the natural world, a cloud passing over the sun may ๆ Present address: School of Psychology, Birmingham University, Edgbaston, Birmingham B15 2TT, UK

\# Present address: Department of Vision Sciences, Aston University, Birmingham B4 7ET, UK 
cause illuminant changes which are almost instantaneous, but it may also cause more gradual changes which take place over several seconds. The purpose in the work reported here was to determine how the ability to discriminate illuminant and spectral-reflectance changes is affected when these changes occur gradually instead of instantaneously. The results may help to clarify the nature of the underlying cue to relational colour constancy.

Such a cue may be available at a relatively low level in the visual system: within any cone class, the ratios of cone excitations produced by light reflected from pairs of different surfaces are almost invariant under a wide range of illuminant changes (Foster and Nascimento 1994). Changes in ratios of cone excitations (or of some related quantities) could provide the signal that the visual system uses to detect changes in reflectance spectra and to discriminate them from illuminant changes: under a spectral-reflectance change there would generally be a large change in these ratios, and under an illuminant change there would generally be a small or zero change in these ratios. If this hypothesis were correct, then the rate at which changes in spectra were made should affect performance; for example, if the changes took place slowly, local receptor adaptation could partly compensate for them, thereby reducing the size of the resulting signal.

Suppose, instead, that the visual system were capable of recovering, at a relatively high level, the spectral reflectances of surfaces, or some colorimetric equivalent (see eg West 1979; Buchsbaum 1980; Worthey 1985; Brill and West 1986; D'Zmura and Lennie 1986; Hurlbert 1986; Maloney and Wandell 1986; Hurlbert and Poggio 1988; Forsyth 1990; D'Zmura and Iverson 1993a, 1993b; Maloney 1993). Then, a change in spectral reflectance might be detected by simply comparing spectral reflectances, or their equivalents, before and after the change; if the change were slow (but not indefinitely long), it should have little effect on performance.

The temporal dependence of relational colour constancy was investigated with a modified version of the paradigm developed by Craven and Foster (1992). Observers were presented with computer simulations of Mondrian patterns of 49 randomly selected Munsell papers. On each experimental trial a Mondrian pattern was presented for $1 \mathrm{~s}$; the pattern was then changed into another pattern, which was also presented for $1 \mathrm{~s}$ and was related to the first either by an illuminant change or by an illuminant change accompanied by changes in the spectral reflectances of individual papers. Illuminant and spectral-reflectance changes were applied linearly with respect to CIE coordinates over temporal intervals ranging from $0 \mathrm{~s}$ (an instantaneous change) to $7 \mathrm{~s}$ (the slowest change). Observers indicated whether there was a spectral-reflectance change.

As the rate of illuminant and spectral-reflectance changes decreased, the duration of a trial increased. To establish whether trial duration was itself important, controls were run in which changes were applied instantaneously but the durations of presentation of the first and second patterns in any trial were each increased so that trial duration was the same as when illuminant and spectral-reflectance changes were applied gradually. A different approach to investigating visual sensitivity to variations in the time course of the illuminant has been described in Nascimento and Foster (1996a).

\section{Method}

\subsection{Apparatus}

Stimuli were generated by an RGB colour-graphics system (Ramtek UK Ltd, Hampshire; 4660 series) under the control of a computer (Sun Microsystems Inc, CA, USA; type 3/160) and displayed on a 19 inch RGB monitor (Sony Corp, Tokyo, Japan; Trinitron GDM-2036S) with a refresh rate of $60 \mathrm{~Hz}$ and phosphor decay time of less than $1 \mathrm{~ms}$. Eight-bit resolution was selected on each gun and screen resolution was 1280 pixels $\times 1024$ pixels. Calibration procedures were as detailed in Nascimento (1995). 


\subsection{Stimuli}

The illuminated Mondrian patterns used as stimuli were all computer-generated images (and any further reference to surfaces, papers, or illuminants used in the experiment applies to these computer simulations). The patterns were square, with side $6 \mathrm{deg}$ at the viewing distance of $200 \mathrm{~cm}$, and were presented in a dark surround. They consisted of 49 rectangular coloured papers with their centres arranged on a square grid. Paper size was selected randomly from the range $0.86 \mathrm{deg}$ to $1.29 \mathrm{deg}$. A ragged edge to the pattern was avoided by displaying only those parts of the pattern that fell within the $6 \mathrm{deg}$ square. The mean luminance of the papers in the images was set at $4 \mathrm{~cd} \mathrm{~m}^{-2}$. Stimuli were viewed in a darkened room.

The 49 papers were randomly selected from the 1200 displayable papers of the Munsell Book of Color (Munsell Color 1976). A new random selection was made in each trial. The spectral reflectances of the papers were taken from a principalcomponents analysis by Parkkinen et al (1989). The illuminants were taken from a principal-components analysis by Judd et al (1964) of daylights, correlated colour temperatures ranging from about 4300 to about $30000 \mathrm{~K}$.

On each trial one Mondrian pattern was presented for $1 \mathrm{~s}$; it then changed either instantaneously or gradually into a second pattern, which was also presented for $1 \mathrm{~s}$. Changes could be of two kinds: illuminant and spectral reflectance. Illuminant changes were applied linearly in time (with respect to CIE coordinates) over five intervals: $0 \mathrm{~ms}$ (more precisely $16.7 \mathrm{~ms}$, an 'instantaneous' change), $200 \mathrm{~ms}, 500 \mathrm{~ms}, 1000 \mathrm{~ms}$, and $7000 \mathrm{~ms}$. Figure la shows the time course of changes for a $1000 \mathrm{~ms}$ change duration. Because illuminants all fell along the daylight locus they can be represented by their CIE $x$-coordinates alone: the illuminant on the Mondrian pattern displayed for the first $1 \mathrm{~s}$ of any trial was always a bluish illuminant with $x$-coordinate 0.25 ; this illuminant then changed into another illuminant with a larger $x$-coordinate. In figure $1 \mathrm{a}$, the heavy continuous line shows a change to a whitish illuminant with $x$-coordinate 0.31 . The size of the illuminant change is $+0.06 x$-coordinate units in the figure, but could be $+0.03,+0.06$ or +0.09 .

Spectral-reflectance changes were simulated by applying different illuminant changes to different papers. For example, the broken lines in figure la show time courses where half the papers in a pattern had an illuminant change of $+0.03 x$-coordinate units applied to them and the other half a change of $+0.09 x$-coordinate units, equivalent to an illuminant change of $+0.06 x$-coordinate units being applied to all papers and an additional nonuniform illuminant change, or what will henceforth be referred to as a reflectance change, of $+0.03 x$-coordinate units being applied to half the papers at random and $-0.03 x$-coordinate units to the other half at random. Across trials, reflectance changes had $x$-coordinates $\pm 0.01, \pm 0.02$, or \pm 0.03 and could occur in all combinations with illuminant changes with $x$-coordinates $+0.03,+0.06$, and +0.09 .

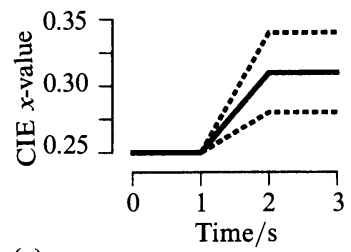

(a)

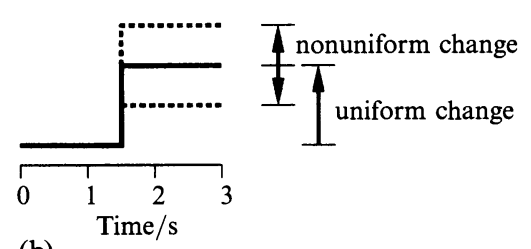

(b)

Figure 1. Time course of change in illuminant CIE $x$-coordinate for (a) a trial with change duration $1000 \mathrm{~ms}$ and (b) a corresponding control trial with the same trial duration but an instantaneous illuminant change. 
Control measurements were made for each of the $500 \mathrm{~ms}, 1000 \mathrm{~ms}$, and $7000 \mathrm{~ms}$ change durations; for example, for the $1000 \mathrm{~ms}$ change duration, the time course for the control was as in figure lb: the first and second Mondrian patterns were each presented for $1500 \mathrm{~ms}$, so the trial duration was $3000 \mathrm{~ms}$, exactly as for the $1000 \mathrm{~ms}$ change duration. Analogously, control measurements for the $500 \mathrm{~ms}$ and $7000 \mathrm{~ms}$ change durations comprised presentations of $1250 \mathrm{~ms}$ and $4500 \mathrm{~ms}$ of the two Mondrian patterns.

\subsection{Design}

Eight different time courses were used in the experiment: five experimental time courses with the five different illuminant and spectral-reflectance change durations ranging from 0 to $7000 \mathrm{~ms}$ and fixed illuminant durations at the beginning and end of every trial of $1000 \mathrm{~ms}$, and three control time courses with change durations of $0 \mathrm{~ms}$ and the three different fixed illuminant durations of 1250,1500 , and $4500 \mathrm{~ms}$. In each session, the time course was held constant, to guard against the tendency (noticed in pilot studies) of observers to describe all changes, whether illuminant or spectralreflectance, as illuminant changes when they were sufficiently slow. Every session comprised 108 trials, in which, in random order, half contained illuminant changes only and the other half contained illuminant and reflectance changes. The eight different time courses were tested in random order across sessions. In total, each time course had eight sessions dedicated to it, so that in the course of the experiment each combination of reflectance and illuminant changes was presented forty-eight times for each of the eight time courses.

\subsection{Procedure}

Observers were given instructions in which they were reminded that the colour of light illuminating our world can change. They were told that in each trial of the experiment one of two stimulus changes would occur: a change in the illuminant, or a change in the illuminant in addition to a change in the spectral reflectances of individual papers, and that their task was to signal whether or not a reflectance change occurred by pressing one of two response buttons. Feedback was only given on the few practice trials which began every session. Observers were not given fixation instructions (there is little evidence for viewing strategies exerting major effects on performance; Cornelissen and Brenner 1995). Before every session, observers were dark adapted for $3 \mathrm{~min}$.

\subsection{Observers}

There were two observers, GP and ME, aged 31 and 21 years, respectively. Each had normal colour vision, as assessed with the Farnsworth-Munsell 100-hue test, and . normal visual (Snellen) acuity. Each was unaware of the purpose of the experiment.

\section{Results}

Discrimination performance of each observer for each combination of reflectance and illuminant changes was quantified in terms of the discrimination index $d^{\prime}$ from signal-detection theory. Thus, for each such combination, let $H$ be the hit rate, that is, the proportion of responses 'reflectance change' in trials containing a reflectance change; let $F$ be the false-alarm rate, that is, the proportion of responses 'reflectance change' in trials not containing a reflectance change; and let $\Phi^{-1}$ be the inverse of the (cumulative) standardised normal distribution. Then the value of $d^{\prime}$ for this combination of changes is given by

$$
d^{\prime}=\Phi^{-1}(H)-\Phi^{-1}(F) .
$$


This function increases monotonically with increasing level of performance, and zero corresponds to chance performance.

Data are plotted in figure 2 for the two observers, ME and GP. There are nine graphs for each observer, in three columns and three rows. Each graph shows $d^{\prime}$ as a function of the duration of reflectance and illuminant changes (solid symbols); control data for the same trial durations but instantaneous changes are also shown (open symbols). The rows of graphs correspond to the three sizes of illuminant changes: $0.03,0.06$, and $0.09 \mathrm{CIE} x$-coordinate units (indicated on the right of the figure). The columns of graphs correspond to the three sizes of reflectance changes: $0.03,0.02$, and $0.01 \mathrm{CIE} x$-coordinate units (indicated along the top of the figure).

The data replicate Craven and Foster's (1992) findings (from their experiment 3) that discrimination improves with increasing magnitude of reflectance change $(0.01$ to $0.03 x$-coordinate units), and worsens with increasing magnitude of illuminant change ( 0.03 to $0.09 x$-coordinate units).

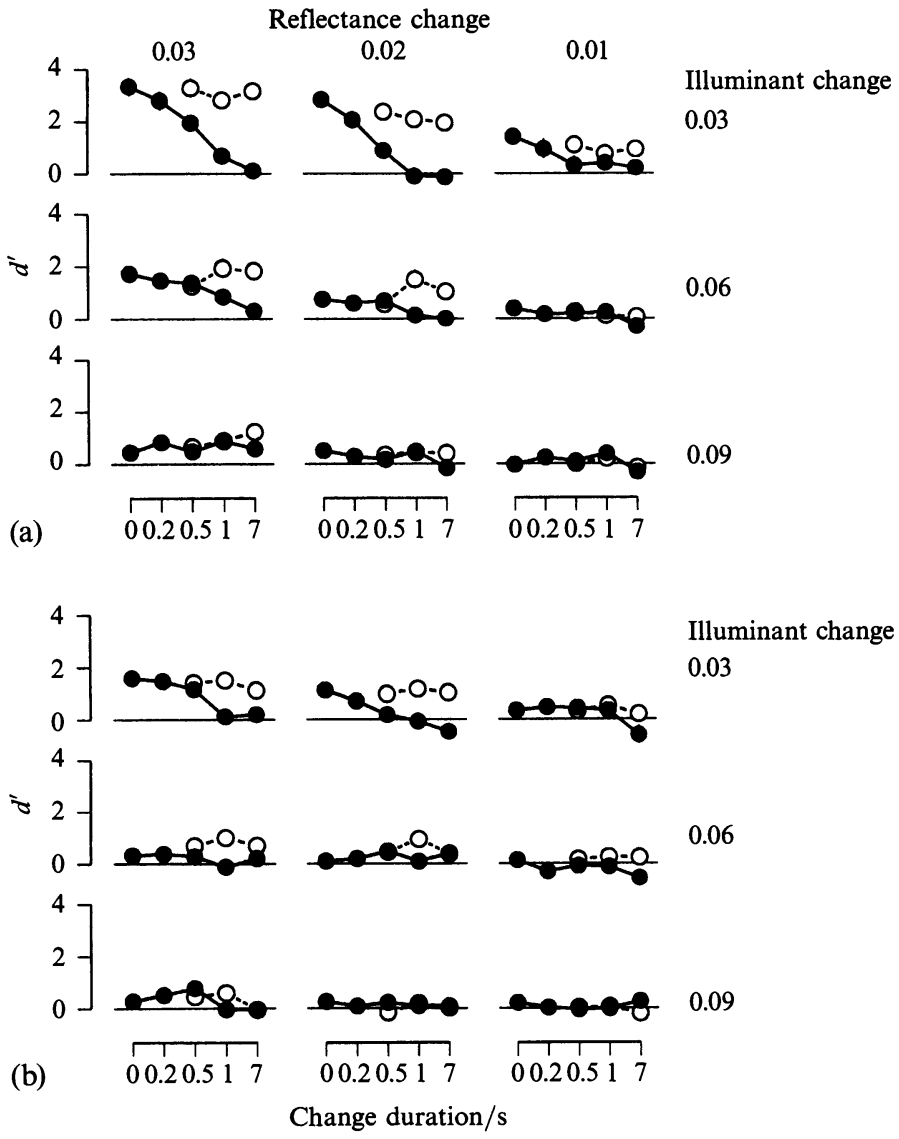

Figure 2. Performance for (a) observer ME and (b) observer GP in discriminating spectralreflectance changes from illuminant changes as a function of the time course of those changes (solid symbols); control data for the same trial durations but instantaneous changes are also shown (open symbols). Discrimination index $d^{\prime}$ from signal-detection theory is plotted against change duration. Vertical bars show \pm 1 SEM, where sufficiently large. Notice that the changeduration axis is nonuniform. The rows of graphs correspond to the three sizes of illuminant changes: $0.03,0.06$ and 0.09 CIE $x$-coordinate units (indicated on right of figure). The columns of graphs correspond to the three sizes of reflectance changes: $0.03,0.02$, and $0.01 \mathrm{CIE} x$-coordinate units (indicated along top of figure). 
A repeated-measures analysis of variance was applied to the two observers' data obtained with the five main change durations: the effect of rate of change was significant $\left(F_{4,4}=6.86, p=0.04\right)$, although there was also a significant interaction between the rate of change and size of illuminant change $\left(F_{8,8}=5.01, p=0.02\right)$. Analysis of the control data and data from the main experiment obtained with instantaneous reflectance and illuminant changes showed that the effect of trial duration was not significant $\left(F_{3,3}=1.34, p=0.41\right)$.

\section{Discussion}

The rate at which illuminant and spectral-reflectance changes occurred significantly affected observers' discriminative ability, but total stimulus (ie trial) duration had little influence, at least over the range of durations considered here. As was suggested in section 1, the effect of time course of illuminant and reflectance changes seems unlikely to be attributable to a simple comparison of recovered spectral reflectances, or their equivalents, before and after the changes. Instead the visual system may use some signal whose magnitude is determined by a change in low-level quantities, such as cone excitation ratios, accompanying spectralreflectance changes.

From figure 2, it is clear that detectability of this hypothesised signal decreased with increasing duration of the reflectance change, that is, with decreasing rate of reflectance change. For both observers, the lowest detectable rate of reflectance change in the presence of the smallest illuminant change was about $0.04-0.06 x$-coordinate units per second (corresponding to a discrimination index $d^{\prime}$ value of about 1.0). Lower rates of change were apparently not detectable. This dependence of the signal on time course, that is, its sensitivity to rate of change and the existence of a lower limit, implies that it is primarily a transient signal.

The level at which this signal originates is not immediately clear. Whilst useful information is potentially available at the receptoral level, it is also available postreceptorally: just as the ratios of cone excitations produced by light reflected from pairs of different surfaces are almost invariant under illuminant changes, so too are the ratios of colour-opponent signals and luminance signals (Nascimento and Foster 1996b). Irrespective of the precise site of origin of the signals they may be transmitted to higher visual centres along luminance or colour-opponent channels, or both.

Some indication of the sensitivity of these signals to channel of transmission in the visual system was obtained by confining the signals either to colour or to luminance channels (Linnell and Foster 1995). Thus, in colour-clamped and luminance-clamped versions of the experiment, the colours and luminances of Mondrian patterns were each constrained throughout any given trial to remain the same. When, for example, luminance was clamped, individual colour patches in the pattern had different colours and luminances; but, although their colours changed during the trial, their luminances did not. It was found that there was no significant interaction between change duration and clamping condition, that is, luminance-clamped or colour-clamped $\left(F_{4,4}=1.11\right.$, $p=0.46$ ) (Linnell and Foster 1995). As discrimination performance with instantaneous changes was only slightly higher than that with changes lasting about $200 \mathrm{~ms}$, the detection mechanisms involved seem unlikely to require the faster response characteristics of a luminance channel (where integration times are of the order of $40 \mathrm{~ms}$ for the range of luminances used here).

In summary, the present findings support the suggestion that discrimination in the tasks considered here depends on the extraction of some low-level transient signal which is generated in response to rapid changes in the spectral reflectances of scenes and which is progressively attenuated as changes occur more and more gradually. 
Although it is uncertain whether this signal originates at receptoral or postreceptoral levels, it does not seem likely that. high-level computations of spectral-reflectance characteristics are required for observers to perceive the material world as changing or stable.

Acknowledgements. This work was supported by the Wellcome Trust (grant no 034807). We thank S M C Nascimento for helpful advice, L M Doherty and C J Savage for critical review of the manuscript, and J P S Parkkinen, J Hallikainen, and T Jaaskelainen for providing their data for the spectral reflectances of the Munsell papers.

\section{References}

Arend L, Reeves A, 1986 "Simultaneous color constancy" Journal of the Optical Society of America A 3 1743-1751

Bramwell D I, Hurlbert A C, 1993 "The role of object recognition in colour constancy" Perception 22 supplement, $62-63$

Brill M H, West G, 1986 "Chromatic adaptation and color constancy: a possible dichotomy" Color Research and Application 11 196-204

Buchsbaum G, 1980 "A spatial processor model for object colour perception" Journal of the Franklin Institute 310 1-26

Cornelissen F W, Brenner E, 1995 "Simultaneous colour constancy revisited: an analysis of viewing strategies" Vision Research $352431-2448$

Craven B J, Foster D H, 1992 "An operational approach to colour constancy" Vision Research $321359-1366$

D'Zmura M, Iverson G, 1993a "Color constancy. I. Basic theory of two-stage linear recovery of spectral descriptions for lights and surfaces" Journal of the Optical Society of America A 10 $2148-2165$

D'Zmura M, Iverson G, 1993b "Color constancy. II. Results for two-stage linear recovery of spectral descriptions for lights and surfaces" Journal of the Optical Society of America A 10 $2166-2180$

D'Zmura M, Lennie P, 1986 "Mechanisms of color constancy" Journal of the Optical Society of America A $31662-1672$

Forsyth D A, 1990 "A novel algorithm for color constancy" International Journal of Computer Vision 5 5-36

Foster D H, Craven B J, Sale E R H, 1992 "Immediate colour constancy" Ophthalmic and Physiological Optics 12 157-160

Foster D H, Nascimento S M C, 1994 "Relational colour constancy from invariant coneexcitation ratios" Proceedings of the Royal Society of London, Series B 257 115-121

Hurlbert A, 1986 "Formal connections between lightness algorithms" Journal of the Optical Society of America A $31684-1693$

Hurlbert A C, Poggio T A, 1988 "Synthesizing a color algorithm from examples" Science 239 $482-485$

Judd D B, MacAdam D L, Wyszecki G, 1964 "Spectral distribution of typical daylight as a function of correlated color temperature" Journal of the Optical Society of America 54 1031-1040

Linnell K J, Foster D H, 1995 "Operational colour constancy under gradual changes of illuminant mediated by colour and luminance channels" Perception 24 supplement, 60

Lucassen M P, Walraven J, 1993 "Quantifying color constancy: evidence for nonlinear processing of cone-specific contrast" Vision Research $33739-757$

Maloney L T, 1993 "Color constancy and color perception: the linear-models framework", in Attention and Performance XIV Eds D E Meyer, S Kornblum (Cambridge: MIT Press) pp 59- 78

Maloney L T, Wandell B A, 1986 "Color constancy: a method for recovering surface spectral reflectance" Journal of the Optical Society of America A 3 29-33

Munsell Color, 1976 Munsell Book of Color-Matte Finish Collection (Baltimore, MD: Munsell Color Corporation)

Nascimento S M C, 1995 Surface Colour Perception under Illuminant Transformations PhD thesis, Keele University, UK

Nascimento S M C, Foster D H, 1996a "Dependence of colour constancy on the time-course of illuminant changes", in John Dalton's Colour Vision Legacy Eds C M Dickinson, I J Murray, D Carden (London: Taylor and Francis)

Nascimento S M C, Foster D H, 1996b "Relational colour constancy and invariant coneexcitation ratios in achromatic and chromatic but isoluminant images" (in preparation) 
Parkkinen J P S, Hallikainen J, Jaaskelainen T, 1989 "Characteristic spectra of Munsell colors" Journal of the Optical Society of America A 6 318-322

Reeves A, 1992 "Areas of ignorance and confusion in color science" Behavioral and Brain Sciences 15 49-50

Tiplitz Blackwell K, Buchsbaum G, 1988 "Quantitative studies of color constancy" Journal of the Optical Society of America A $51772-1780$

Troost J M, Weert C M M de, 1991 "Naming versus matching in color constancy" Perception \& Psychophysics $50591-602$

Valberg A, Lange-Malecki B, 1990 "Colour constancy in Mondrian patterns: a partial cancellation of physical chromaticity shifts by simultaneous contrast" Vision Research 30 371-380

West G, 1979 "Color perception and the limits of color constancy" Journal of Mathematical Biology 8 47-53

Worthey A, 1985 "Limitations of color constancy" Journal of the Optical Society of America A $21014-1026$ 